\title{
Terminal Arbors of Axons Projecting to the Somatosensory Cortex of the Adult Rat. II. The Altered Morphology of Thalamocortical Afferents Following Neonatal Infraorbital Nerve Cut
}

\author{
Karl F. Jensen ${ }^{1}$ and Herbert P. Killackey ${ }^{2}$ \\ 1.2Department of Psychobiology and 'Department of Anatomy and Neurobiology, University of California, Irvine, California \\ 92717; and 'Division of Neurotoxicology, Health Effects Research Laboratory, U.S. Environmental Protection Agency, \\ Research Triangle Park, North Carolina 27711
}

The organization of the whisker representation within the neocortex of the rat is dependent on an intact periphery during development. To further investigate how alterations in this cortical map arise we examined the organization of thalamocortical afferents to the whisker representation in adult animals in which the infraorbital branch of the trigeminal nerve was cut on the day of birth. The disrupted pattern of thalamocortical projections to the vibrissae representation was apparent in the abnormal pattern of the anterograde transport of horseradish peroxidase from the thalamus, as well as in the abnormal pattern of succinate dehydrogenase activity. To determine the morphology of individual thalamocortical axons associated with this disrupted pattern, terminal arbors were "bulk-labeled" by injections of horseradish peroxidase into the white matter beneath the somatosensory cortex. Terminal arbors were identified by their laminar distribution of boutons corresponding to the specific thalamocortical afferent. The medial to lateral extent of these terminal arbors varied dramatically, from 350 to $1500 \mu \mathrm{m}$. In addition, terminal arbors innervating the same local area of cortex appeared to have varying degrees of overlap. Thus, the disruption of the neocortical vibrissae representation appears to involve the abnormal arborization of individual thalamocortical afferents. This finding supports the hypothesis that the fine-grain organization of the somatotopic map is dependent on the morphology and organization of individual thalamocortical arbors, which, in turn, are dependent on the periphery during development.

Representation of the sensory periphery is a fundamental characteristic of the neocortex. The role of the periphery in establishing a neocortical map is a central issue in neocortical organization (Van der Loos and Woolsey, 1973). The discrete organization of the vibrissae representation lends itself to the investigation of mechanisms by which the periphery influences

\footnotetext{
Received June 30, 1986; revised May 26, 1987; accepted May 26, 1987.

This work was supported by NSF Grant 84-18715 to H.P.K. and NIH Postdoctoral Fellowship NSO6651 to K.F.J.

This manuscript has been reviewed by the Health Effects Research Laboratory, U.S. Environmental Protection Agency, and approved for publication. Mention of trade names or commercial products does not constitute endorsement or recommendation for use.

Correspondence should be addressed to Dr. Karl F. Jensen, Neurotoxology Division, MD 74-B, Health Effects Research Laboratory, U.S. Environmental Protection Agency, Research Triangle Park, NC 27711.

Copyright $(1) 1987$ Society for Neuroscience $0270-6474 / 87 / 113544-10 \$ 02.00 / 0$
}

neocortical organization. In particular, the organization of thalamocortical projections to the rodent somatosensory cortex is dependent on the integrity of the innervation of the facial whiskers during early neonatal development (Killackey and Belford, 1979). When peripheral damage is selective, the altered pattern of thalamocortical projections closely parallels the damage to the peripheral pattern of innervation (Killackey et al., 1976; Killackey and Belford, 1980; Durham and Woolsey, 1984).

However, the question remains, To what extent does the altered pattern of thalamocortical projections result from changes in individual terminal arbors? In the previous paper, we demonstrated that the size of an individual thalamacortical terminal arbor corresponds to the size of the neocortical representation of a single vibrissa (Jensen and Killackey, 1987). In the present study, we examined individual thalamocortical terminal arbors in rats in which the infraorbital nerve, which innervates the vibrissae follicles, was cut at birth. We focused on the morphology of these terminal arbors to evaluate how neonatal peripheral alterations affect the arborization patterns of individual thalamocortical axons.

Abstracts of this work were presented at the Society of Neuroscience Meetings in in 1983, 1984, and 1985.

\section{Materials and Methods}

The results of this study were based on observations in 52 adult SpragueDawley rats. In 46 of these rats, the infraorbital branch of the right trigeminal nerve was cut at birth. The organization of thalamocortical projections was analyzcd in 3 ways. First, to demonstrate the vibrissaerelated pattern, $60 \mu \mathrm{m}$ sections of flattened neocortical hemispheres were stained for the activity of succinate dehydrogenase. Second, to demonstrate the overall pattern of thalamocortical projections, large injections of WGA-HRP $(0.25-0.50 \mu \mathrm{l}, 20 \%$ in water) were made into the ventral posterior region of the thalamus from a horizontal posterior approach through the cerebellum. The injections were made along a single anteroposterior trajectory sufficient to anterogradely label the posteromedial aspects of the primary somatosensory cortex (SmI). Following a survival time of $24 \mathrm{hr}$, the rats were perfused according to the method of Rosene and Mesulam (1978). The $100 \mu \mathrm{m}$ sections of flattened cortex were processed with tetramethylbenzidine. Third, to demonstrate the morphology of individual terminal arbors, axons were "bulklabeled" from injections of WGA-HRP into the internal capsule below the somatosensory cortex. Individual terminal arbors were reconstructed from serial sections. "Specific" thalamocortical terminal arbors were identified as having the majority of their branches and boutons in layer IV. The size and branching density of the layer IV plexus of the reconstructed terminal arbors was also estimated. Since the overall mediolateral extent of the terminal arbors was, in some cases, much greater than the mediolateral extent in any given section, the mediolateral dimension 
was averaged across all sections to avoid overestimating the projection area. The approximate location and size of the reconstructed terminal fields were also plotted on a surface view of the somatosensory neocortex for comparison with the distribution of succinate dehydrogenase clusters. The above procedures are described in detail in the previous paper (Jensen and Killackey, 1987).

\section{Results}

\section{Alterations in the pattern of thalamocortical projections}

The adult pattern of thalamocortical projections to the somatosensory cortex was characterized in 2 ways. First, the pattern of succinate dehydrogenase activity in tangential sections of flattened neocortex, which has been previously shown to correspond to the pattern of thalamocortical afferents (Killackey and Belford, 1979), was examined in normal animals and in animals in which the infraorbital nerve was cut at birth. Second, the pattern of anterograde label following large injections of WGA-HRP into the thalamus was also examined in tangential sections of flattened neocortex from normal animals and from animals in which the infraorbital nerve was cut at birth.

The pattern of succinate dehydrogenase activity in tangential sections from adult animals in which the infraorbital nerve was sectioned at birth was significantly altered from the normal pattern (Fig. 1). In normal animals, discretely organized patches of succinate dehydrogenase activity replicate the arrangement of vibrissae on the face. Five rows of 4-6 patches each exhibit an anterolateral to posteromedial orientation. In addition, a posterior row of 4 large patches exhibits an anteromedial to posterolateral orientation.

In animals in which the infraorbital nerve was cut at birth, the most striking alteration is in the region of the vibrissae representation. Alterations in vibrissae-related clustering are apparent in flattened sections of neocortex stained for succinate dehydrogenase activity. Patches of stain, variable in size and shape, appear in the posteromedial region normally occupied by the 5 rows of vibrissae-related clusters. In the more anterolateral region of the vibrissae representation, these abnormal patches are oriented longitudinally in the anteroposterior direction and separated by lighter-staining areas. In the most posteromedial region of the vibrissae representation, the patches appear denser and more tightly packed than in the anterolateral region. In addition to alterations in the region of the mystacial vibrissae representation, the organization of patches in the region of the anterior sinus hair representation is also altered. Patches of irregular size and shape are apparent in this region.

The pattern of anterograde label following large injections of the WGA-HRP in the ventral posterior region of the thalamus largely coincide with the above-described pattern of succinate dehydrogenase activity (Fig. 2). In normal animals, clusters of anterograde label are arranged in a pattern that replicates the appearance of the body surface. Within the region of the vibrissae representation there are 5 rows of 4-6 dense clusters each, arranged in an anterolateral to posteromedial orientation. In addition, there is a posterior row of 4 large clusters arranged in an anteromedial to posterolateral orientation. The arrangement of these clusters corresponds to the arrangement of mystacial vibrissae. The dense clusters of the vibrissae representation contrast with the more homogeneous appearance of the region corresponding to the trunk representation (Fig. 2C).

In sections of the neocortex from adult animals in which the infraorbital nerve was cut at birth (Fig. 2, $A, B$ ), there are irregularly shaped, dense clusters of label that are tightly packed
Table 1. Dimensions of the layer IV plexuses of individual terminal arbors

\begin{tabular}{cclll} 
Figure & $\begin{array}{l}\text { Branch (b) } \\
\text { density } \\
\left(\mathrm{b} / \mathrm{mm}^{3}\right)\end{array}$ & $\begin{array}{l}\text { Medio- } \\
\text { lateral } \\
\text { extent } \\
(\mathrm{mm})\end{array}$ & $\begin{array}{l}\text { Antero- } \\
\text { posterior } \\
\text { extent } \\
(\mathrm{mm})\end{array}$ & $\begin{array}{l}\text { Projection } \\
\text { area } \\
\left(\mathrm{mm}^{2}\right)\end{array}$ \\
\hline 3 & 9 & 1.50 & 1.45 & 0.78 \\
4 & 113 & 0.75 & 0.30 & 0.09 \\
5 & 299 & 0.35 & 0.25 & 0.06 \\
$6 \mathrm{a}$ & 29 & 0.85 & 0.70 & 0.20 \\
$6 \mathrm{~b}$ & 235 & 0.50 & 0.30 & 0.08 \\
7 & 190 & 0.43 & 0.45 & 0.10 \\
8 & 41 & 0.70 & 1.25 & 0.31 \\
9 & 33 & 0.90 & 0.75 & 0.18 \\
10 & 51 & 0.65 & 0.40 & 0.07 \\
Mean \pm SE & $111 \pm 33$ & $0.74 \pm 0.11$ & $0.65 \pm 0.14$ & $0.21 \pm 0.08$ \\
\hline
\end{tabular}

within the posteromedial aspect of the face representation. In the anterolateral aspect of the face region, where 5 rows of clusters normally occur, there are elongated, irregular stripes oriented in the anterolateral direction. Associated with these stripes are dense, amorphous patches of variable size.

Thus, the pattern of anterograde label in the region of somatosensory cortex corresponding to the mystacial vibrissae representation is dramatically altered in adult animals in which the infraorbital nerve was cut at birth. This abnormal pattern appears to have 2 significant features: densely packed clusters in the posteromedial region and elongated stripes of varying density in the anterolateral region.

\section{Morphology of afferent terminal arbors}

WGA-HRP was injected into the white matter beneath the somatosensory cortex in animals in which the infraorbital nerve was cut at birth in order to examine the morphology of the terminal arbors associated with the abnormal pattern of thalamocortical projections. Terminal arbors that had the majority of their boutons in layer IV were labeled. When the parent fiber of these terminal arbors was seen arising from the white matter, it followed a tortuous course through the deeper cortical layers (Figs. 3-6). Parent fibers could be seen to have both small- and large-caliber collaterals in layer VI (Figs. 3, 4, 6, 7). Large-caliber collaterals could be seen giving rise to a few fine branches in layer V. Some of the small-caliber fibers arborized slightly after branching from the parent fiber in the deeper layers. In a number of cases, large-caliber collaterals could be seen to traverse a significant distance in the medial to lateral dircetion. Observations of serial sections indicated that these large-caliber branches could traverse over $1 \mathrm{~mm}$ in the anteroposterior direction (Figs. 3, 8, Table 1). Large-caliber collaterals tend to branch in the upper part of layer $\mathrm{V}$, giving rise to a plexus in layer IV. Some collaterals of the layer IV plexus extend into layer III, and a smaller number extend into layer II.

Boutons were observed on most of the fine-caliber collaterals. The greatest density of boutons occurs in layer IV. The collaterals extending from this plexus have boutons in layers III and II. The fine collaterals in layer VI and, to a lesser extent, those in $\mathrm{V}$, also display a number of boutons.

There is significant variability in the dimensions of the layer IV plexus. The arbors having a dense layer IV plexus (Table 1, Figs. 4-7, 1 b) extend $350-750 \mu \mathrm{m}$ in the mediolateral dimen- 

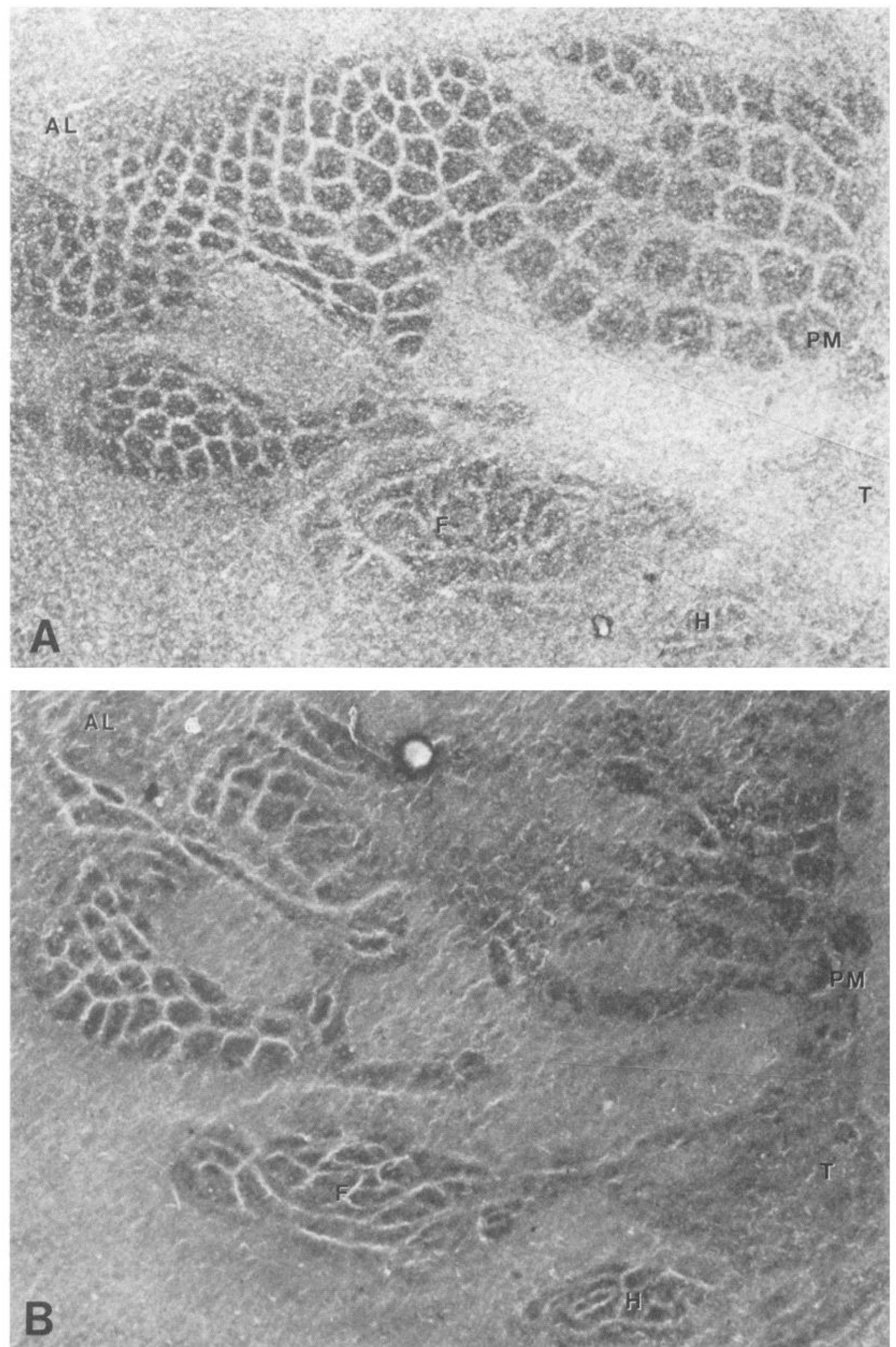

Figure 1. A, Photomontage of sections of flattened cortex stained for succinate dehydrogenase activity from a normal adult animal. $P M$, Posteromedial aspect of mystacial vibrissae representation. $A L$, Anterolateral aspect of the anterior sinus representation. $F$, Forepaw representation. $H$, Hindpaw representation. $T$, Trunk representation. Scale bar, $100 \mu \mathrm{m}$. B, Photomontage of sections of flattened cortex stained for succinate dehydrogenase activity from an adult animal in which the infraorbital nerve was cut at birth. Labels and scale as in $A$. 
sion and 250-450 $\mu \mathrm{m}$ in the anteroposterior dimension. Terminal arbors having a sparser layer IV plexus (Table 1; Figs. 3, $8-10,11 \mathrm{~A}$ ) extend $650-1500 \mu \mathrm{m}$ in the mediolateral dimension and $400-1450 \mu \mathrm{m}$ in the anteroposterior dimension. This mixed arrangement of terminal arbors occurs along with varying degrees of overlap between arbors. Even arbors with a layer IV plexus that was relatively restricted in the horizontal dimension could have adjacent terminal fields with little or no overlap (Fig. 6).

\section{Relationship between projection area and branch density}

In animals in which the infraorbital nerve was cut, the projection area of the layer IV plexus ranges from 0.06 to $0.78 \mathrm{~mm}^{2}$ (Table 1). Thus, the sizes of the layer IV plexuses of terminal arbors from the vibrissae region were larger, and individual values varied to a much greater extent than was observed in normal animals (0.02-0.21 $\mathrm{mm}^{2}$; Jensen and Killackey, 1987). Furthermore, terminal arbors from animals in which the infraorbital nerve was cut have, on the average, only $25.5 \%$ of the density of branches of terminal arbors from the vibrissae region of normal animals (normal: $436 \pm 62$ branches $/ \mathrm{mm}^{3}$; nervecut: $111 \pm 33$ branches $/ \mathrm{mm}^{3}$ ). In a manner similar to that reported for normal animals, the relationship between branch density and projection area of the layer IV plexus of the reconstructed terminal arbors can be described by the hyperbolic function:

$$
\text { Branch density }=6.76 *(\text { projection area })^{-1.18} \text {. }
$$

A $\log -\log$ transformation yields a linear model $(p<0.01)$ :

$\log ($ branch density $)=-1.18 \cdot \log ($ projection area $)+0.83$.

The higher-order quadratic and cubic models are not significant $(p>0.8)$. Furthermore, the log of the branch density is negatively correlated with the log of the projection area $(r=-0.86$, $p<0.01$; Fig. 12). A comparison on the regression plot for normal layer IV plexuses (Fig. 12, solid line; replotted from Jensen and Killackey, 1987) with the plot for those from animals with neonatal infraorbital nerve cut (Fig. 12, dotted line) indicates that for layer IV plexuses of the same size, those in the animals in which the infraorbital nerve was cut at birth have a lower density of branches than those in normal animals.

\section{Discussion}

The present results demonstrate that neonatal infraorbital nerve cut alters the organization of afferent projections to the somatosensory neocortex. We have examined 2 characteristics of the altered thalamocortical projection: the overall vibrissae-related pattern and the morphology of individual terminal arbors. Our observations indicate that the changes in the overall pattern of thalamocortical projections involves alterations in morphology of individual terminal arbors.

\section{Relationship between the morphology of individual terminal arbors and the overall pattern of thalamocortical projections}

The overall pattern of thalamocortical projections to the somatosensory neocortex in rats in which the infraorbital nerve was cut at birth indicates that the orientation and relative position of the representation of the major body regions are, to a large extent, normal. This is in line with physiological maps of the neocortex obtained from adult animals in which the infraorbital nerve was cut at birth (Waite and Cragg, 1982; Waite, 1984). The preservation of the gross topography of the somato-
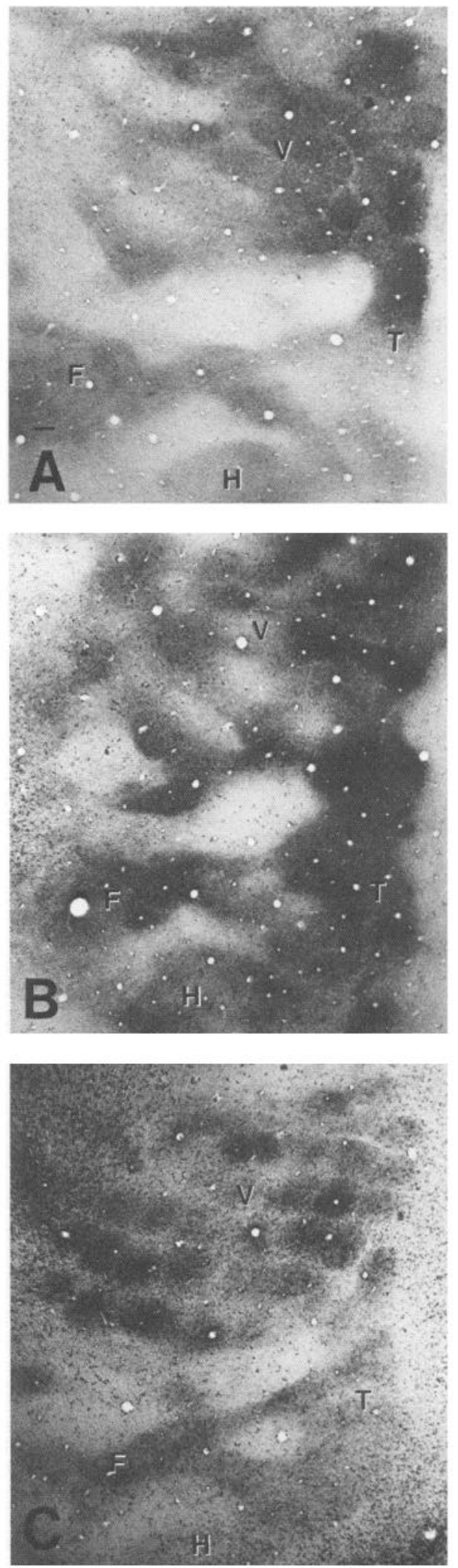

Figure 2. $A$ and $B$, Photomicrographs of sections of flattened cortex demonstrating anterogradely transported HRP from injections of the ventral posterior thalamus in adult animals in which the infraorbital nerve was cut at birth. Scale bar, $100 \mu \mathrm{m}$. $V$, Mystacial vibrissae representation. $F$, Forepaw representation. $H$, Hindpaw representation. $T$, Trunk representation. Scale bar, $100 \mu \mathrm{m}$. $C$, Photomicrograph of a section of flattened cortex demonstrating anterogradely transported HRP from an injection in the ventral posterior thalamus in a normal adult animal. Tetramethylbenzidine reaction. Labels and scale as in $A$. 
Figure 3. Terminal arbor from the anterolateral region of the mystacial vibrissae representation. The medial to lateral extent of the layer IV plexus is $1500 \mu \mathrm{m}$. The view is reconstructed from coronal sections. The top line represents the pia. The bottom line represents the white matter of the internal capsule. Triangles indicate the border of layers III and IV. Circles indicate the border of layers IV and V. Squares indicate the border of layers $\mathrm{V}$ and VI. Diaminobenzidine reaction. Scale bar, $100 \mu \mathrm{m}$.
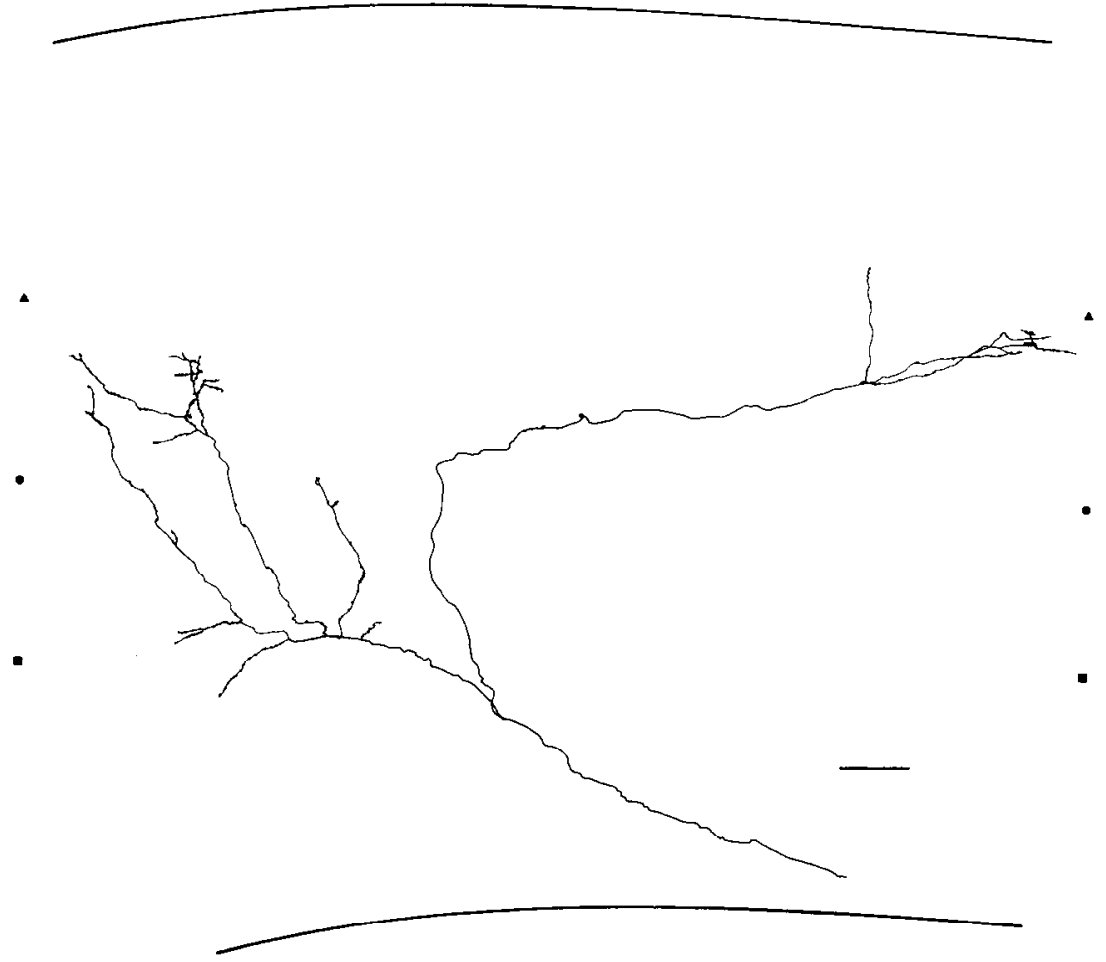

sensory region is consistent with the observation that thalamocortical projections are organized in a topographic manner at birth, at the time when the infraorbital nerve was cut (Dawson and Killackey, 1985).

While the gross topographic organization of the thalamocortical projection is preserved, the somatotopic organization within the face representation is disrupted. In contrast to the normal clustered pattern of thalamocortical projections in the vibrissae region, the altered pattern is composed of elongated stripes and amorphous patches. Several alterations in the morphology of the terminal arbors appear to contribute to the altered pattern. First, terminal arbors are larger and of more varied size than normal. Second, the arbors tend to be elongated in an orientation corresponding to the orientation of the stripes. Third, the extent of overlap between adjacent arbors is highly variable, in sharp contrast to the coextensive terminal fields of normal vibrissae-related terminal arbors. These altered characteristics of thalamocortical afferent morphology are consistent with relatively large numbers of neurons responsive to more than one vibrissa in adult animals in which the infraorbital nerve was cut at birth (Waite and Cragg, 1982; Waite, 1984). Thus, we conclude that the disruption of the neocortical vibrissae map involves a disruption of the normal clustering of thalamocortical afferents.

\section{Effect of nerve cut on the projection area of the layer IV plexus}

The larger than normal projection areas of the layer IV plexuses following neonatal infraorbital nerve cut may occur in either of 2 ways. First, in normal animals, axons may initially branch over an extensive area, and their restricted arbors result from a subsequent retraction of more distant processes. Neonatal damage to the afferent pathways may interfere with the retraction process, resulting in elongated arbors. This possibility has been suggested to account for the disruption of the segregation of ocular dominance columns in the visual cortex following visual deprivation during postnatal development (Levay et al., 1980). Alternatively, normal axons may initially form extensive branches in a restricted field. Neonatal damage to the afferent pathways then reduces the number of branches formed and the axons grow beyond their normally restricted field. This possibility has been proposed to account for enlarged arbors of retinogeniculate axons following neonatal enucleation (Robson et al., 1978). The present observations do not provide conclusive evidence that distinguishes between these 2 possibilities. Several lines of evidence, however, suggest that neonatal infraorbital nerve cut alters the initial growth of thalamic axons into the neocortex rather than a later remodeling process. First, the larger, abnormal terminal arbors in rats with neonatal nerve cut have a lower than normal density of branches. It is therefore unlikely that the abnormal arbors retained branches that are normally lost. Second, the pattern of afferent clusters within the cortex does not appear to arise from a previously diffuse pattern (Killackey and Belford, 1979). Third, thalamocortical axons do not appear to have extensive penetration into the cortical gray matter at birth, but appear to subsequently invade layer IV on postnatal days 2 and 3 (Wise and Jones, 1978). This time period of afferent growth corresponds to the time at which the vibrissaerelated pattern first becomes visible (Killackey and Belford, 1979).

The hypothesis that the clustering of terminal fields of vibrissae-related afferents occurs during the initial growth of thalamic axons contrasts with the hypothesis that the segregation of eye-specific afferents in the visual system results from the remodeling of afferent arbors after initial ingrowth (Levay et al., 1978,1980 ). The different mechanisms that predominate during the patterning of afferent axonal arbors in these 2 systems may be related to differences both in organization and development. The neocortical visual map requires the alignment of afferents from both the ipsilateral and contralateral eyes to form a continuous map of the visual field. Such alignment could result 

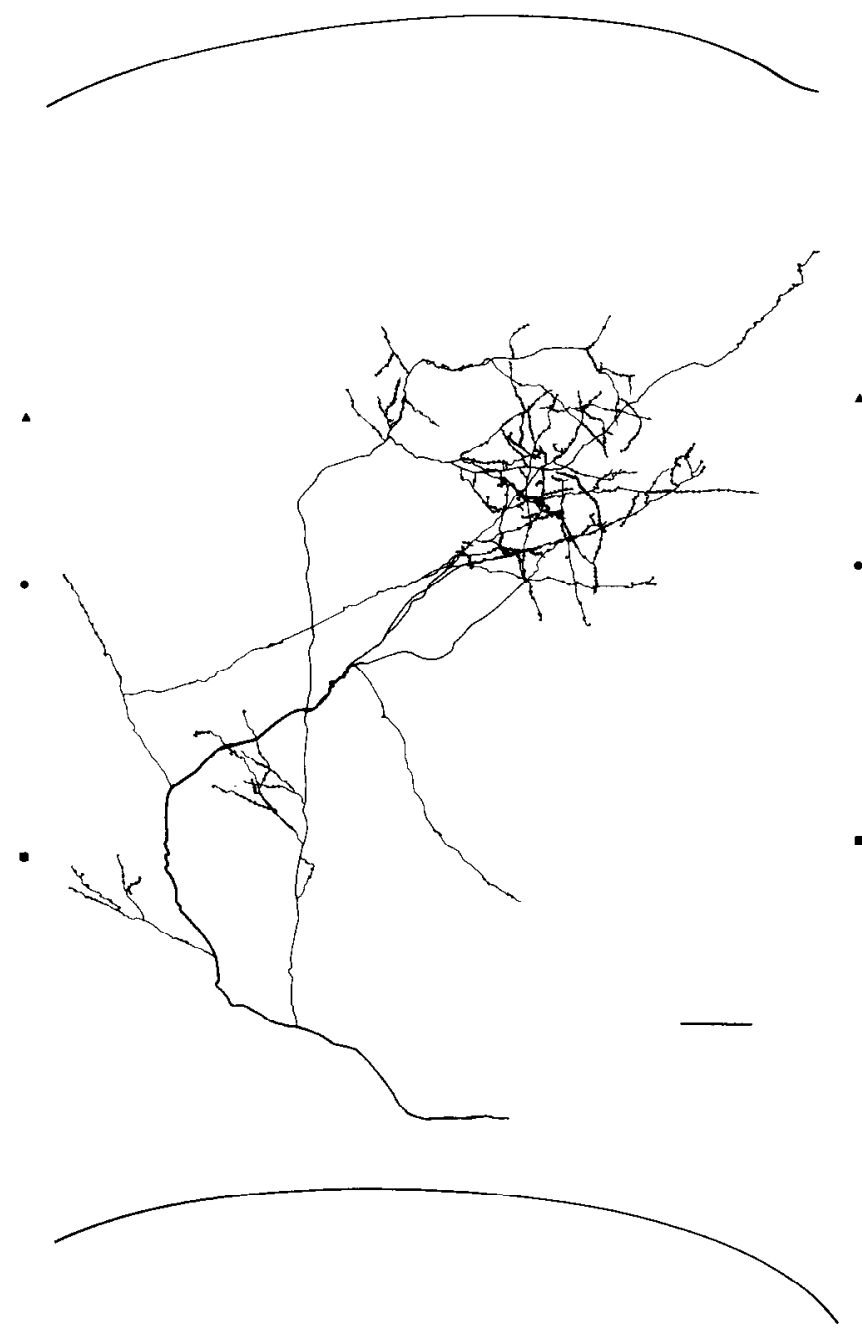

Figure 4. Terminal arbor from the posteromedial region of the mystacial vibrissae representation. The medial to lateral extent of the layer IV plexus is $750 \mu \mathrm{m}$. Details as in Figure 3.

from the loss of collaterals during the formation of interdigitated tufts of eyc-spccific afferents. In contrast, the vibrissa map is characterized by the discrete representation of contralateral receptors and does not appear to involve the interdigitated arbors characteristic of eye-specific afferents. Thus, the dominance of contralateral input to this map may minimize the importance of the loss of long collaterals during pattern formation. Furthermore, in the lateral geniculate nucleus, where eye-specific terminal arbors are discretely organized into different layers, the development of the segregated pattern does not appear to involve the loss of long collaterals (Sretavan and Shatz, 1984). Moreover, enucleation during development appears to alter the elaboration of retinogeniculate terminal arbors rather than reduce the retraction of long collaterals (Robson et al., 1978; Sretavan and Shatz, 1986). Thus, when sensory maps involve the segregation of terminal arbors, the progressive elaboration of axons may dominate the formation of peripherally dependent patterns of afferent innervation.

\section{Relationship between the projection area and branching density of the layer $I V$ plexus}

In animals in which the infraorbital nerve was cut at birth, terminal arbors with smaller projection areas have a greater

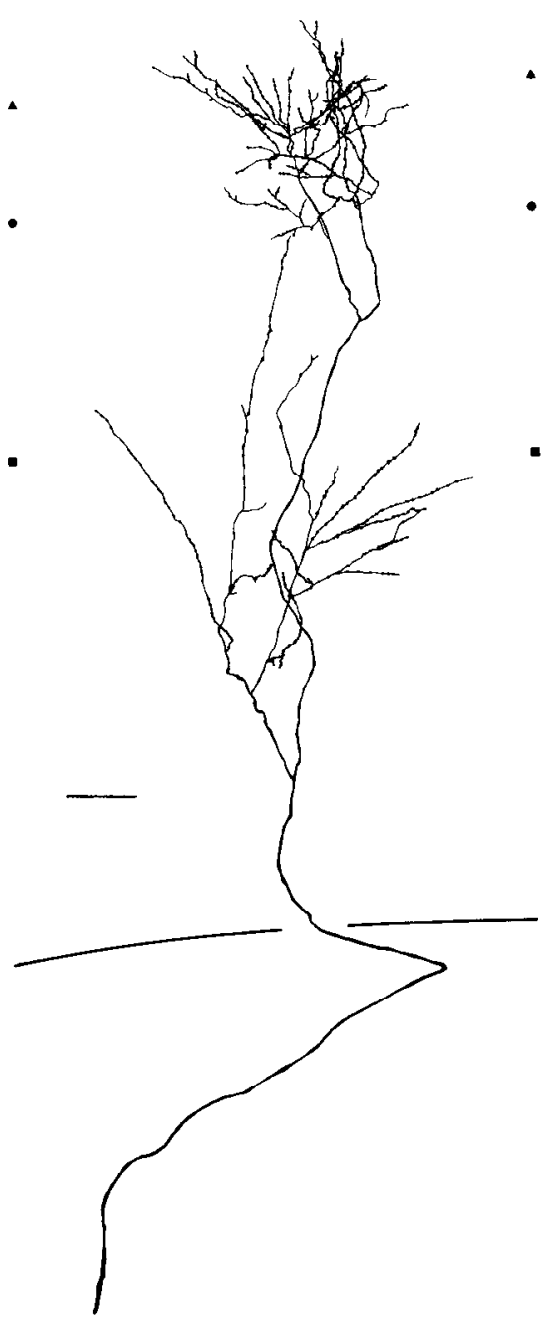

Figure 5. Terminal arbor from the posteromedial region of the mystacial vibrissae representation. The medial to lateral extent of the layer IV plexus is $350 \mu \mathrm{m}$. Details as in Figure 3.

density of branches than those with larger projection areas. The same basic relationship is also true of thalamocortical arbors from normal animals (Jensen and Killackey, 1987). This observation supports the hypothesis that the relationship between branch density and projection area is, to at least some degree, intrinsically regulated by thalamic neurons. However, the actual size and density of the layer IV plexus is dramatically altered by neonatal damage to the infraorbital nerve. Neonatal nerve cut results in larger projection areas and lower branching density. This result indicates that peripheral influences play a significant role in determining the branching density and size of the layer IV plexus of thalamocortical axons.

How does neonatal damage to the peripheral innervation of the vibrissae reduce the branching density and increase the size of the layer IV plexus of thalamocortical axons? Several possibilities are suggested by the distribution of clusters of different sizes in the region of the neocortical mystacial vibrissae representation. Small, tightly packed clusters predominate in the 

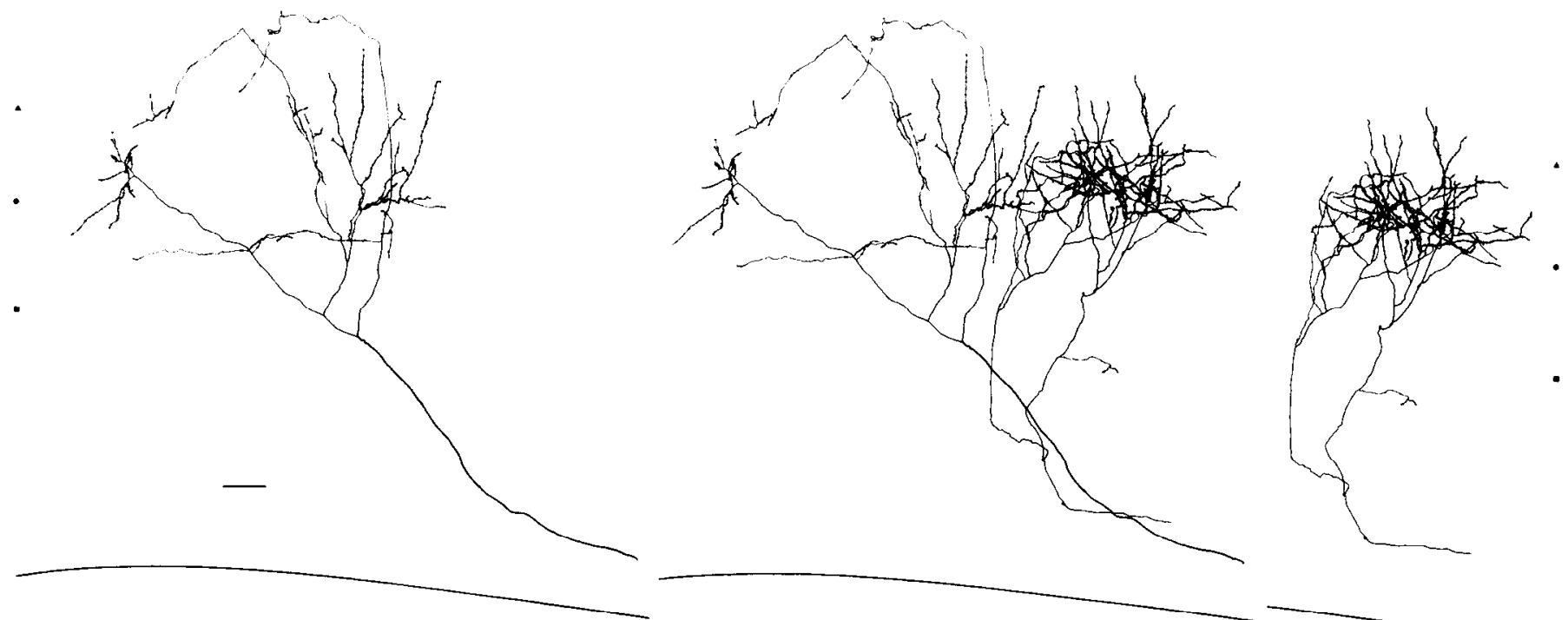

Figure 6. Two terminal arbors that innervate the same localized area of layer IV in the anterolateral region of the mystacial vibrissac representation (Fiber $6 \mathrm{a}$ is to the left, fiber $6 \mathrm{~b}$ is to the right.) Their terminal fields within layer IV do not have an extensive degree of overlap. Details as in Figure 3.

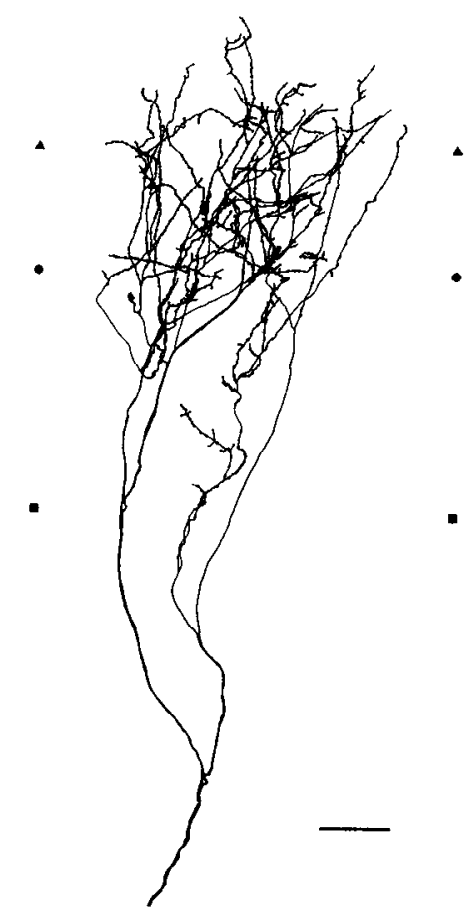

Figure 7. Terminal arbor from the region of somatosensory cortex corresponding to the anterolateral portion of the face representation in an adult animal in which the infraorbital nerve was cut at birth. The medial to lateral extent of the layer IV plexus of this arbor is $430 \mu \mathrm{m}$. Details as in Figure 3.

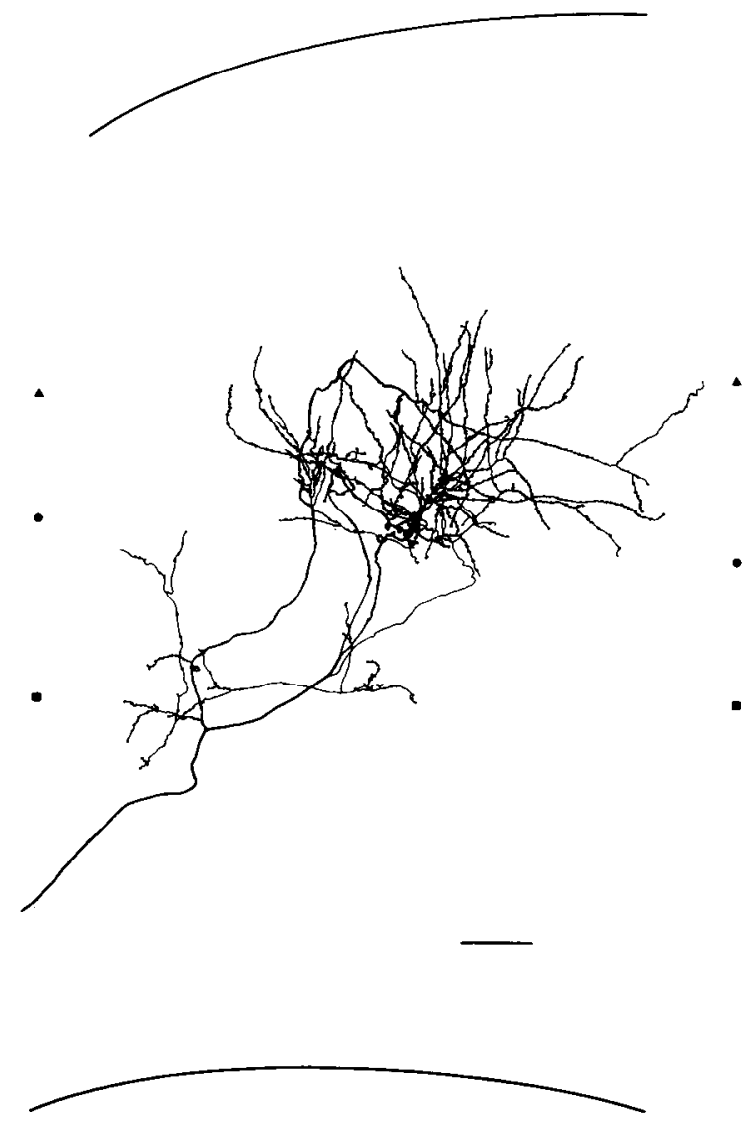

Figure 8. Terminal arbor from the anterolateral region of the mystacial vibrissae representation. The medial to lateral extent of the layer IV plexus is $700 \mu \mathrm{m}$. Details as in Figure 3. 

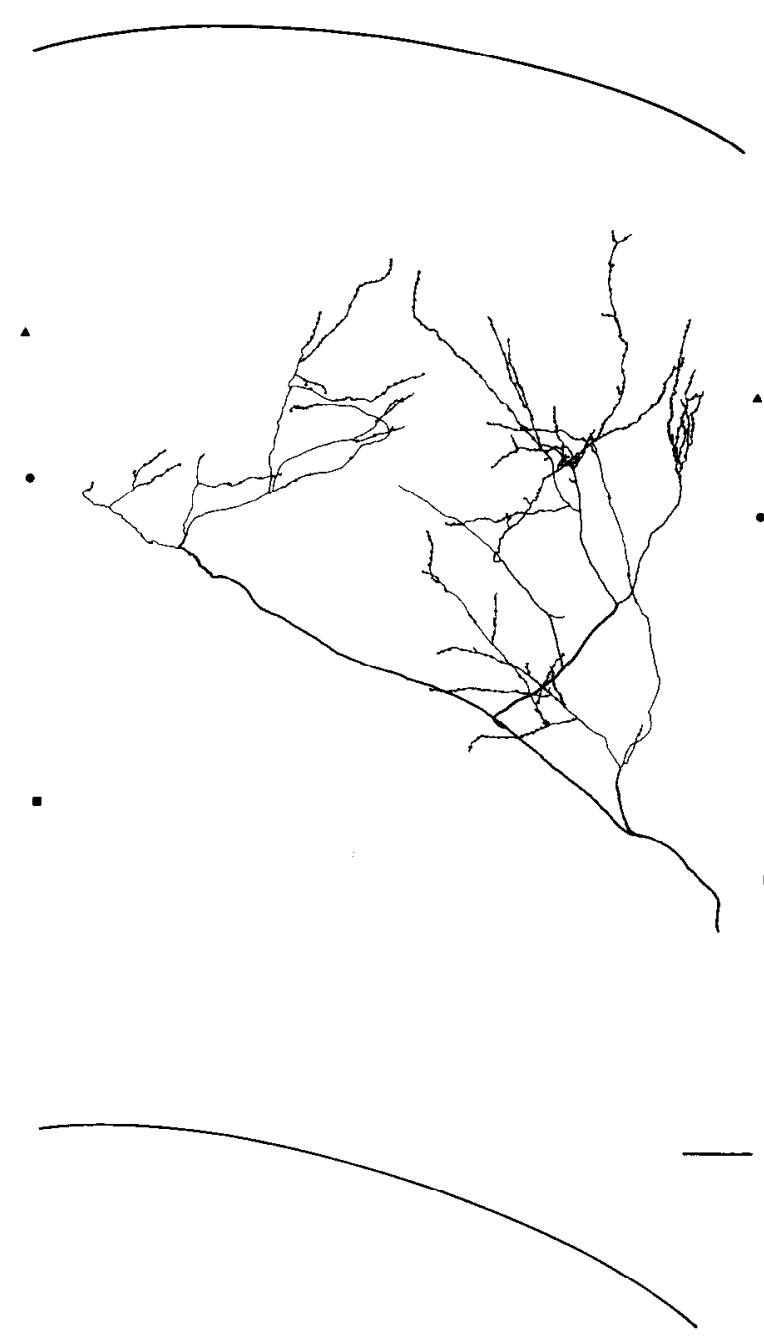

Figure 9. Terminal arbor from the anterolateral region of the mystacial vibrissae representation. The medial to lateral extent of the layer IV plexus is $900 \mu \mathrm{m}$. Details as in Figure 3.

posteromedial region, while elongated stripes are present in the anterolateral region. These regional characteristics could reflect the state of development of the trigeminal pathway at the time the infraorbital nerve was cut. In the brain stem trigeminal complex, the clustered pattern of primary afferents in the ventrolateral principal sensory nucleus is apparent at birth, while the clustered afferents in the dorsomedial principal sensory nucleus is not apparent until several days later (Erzurumlu and Killackey, 1983). Neonatal damage to the vibrissae innervation destroys the associated primary trigeminal afferents (Bates and Killackey, 1985; Jacquin and Rhoades, 1985) and prevents the normal clustering of their postsynaptic neurons in the brain stem trigeminal complex (Bates et al., 1982). Thus, sectioning the entire infraorbital nerve at birth would be expected to have a more profound effect on the representation of the anterior than on the posterior mystacial vibrissae. Densely branched, restricted arbors may reflect the more mature state of innervation of the ventrolateral region of the trigeminal principal sensory nuclcus at birth. Conversely, sparsely branched, elongated arbors may reflect the less mature state of the innervation of the dorsomedial region of the principal sensory nucleus at birth.

A second possibility is that, in the absence of normally clus-

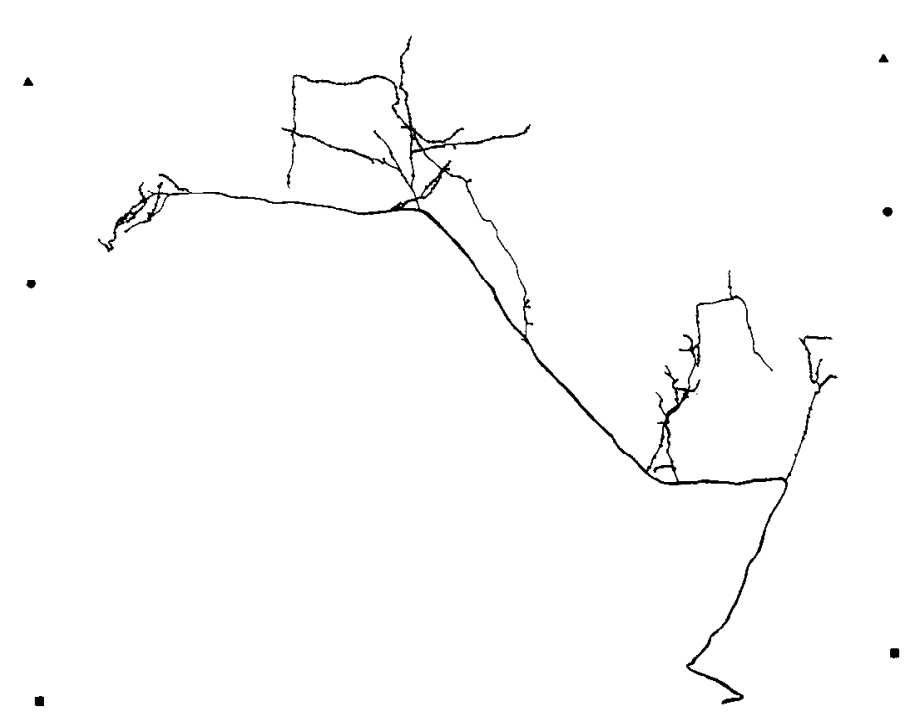

Figure 10. Terminal arbor from the posteromedial region of the vibrissae representation. The medial to lateral extent of the layer IV plexus is $650 \mu \mathrm{m}$. Details as in Figure 3.

tered trigeminothalamic projections, abnormal thalamocortical terminal arbors result from the influence of other sources of afferents to the ventroposterior nucleus. We consider this possibility unlikely, however, since neonatal infraorbital nerve cut results in a uniform pattern in the thalamus, rather than an aberrant one, at a time when normal vibrissae-related clustering is obvious (Killackey and Shinder, 1981). We cannot, however, rule out the possibility that competitive interactions from other neocortical afferents may contribute to the altered pattern. Afferents from the medial aspect of the posterior thalamic nuclear group (Koralek et al., 1985) may compete with those from the ventroposterior nucleus for postsynaptic sites. Damage to the trigeminal pathway may selectively impair the competitive ability of afferents from the ventroposterior nucleus. Such an interaction is likely to be analogous to competitive interactions between $\mathrm{X}$ and $\mathrm{Y}$ retinogeniculate projections. The relative alterations in the $\mathrm{X}$ - and $\mathrm{Y}$-terminal arbors occur significantly later in development than the ingrowth of retinal afferents or their segregation (Sur et al., 1984). Thus, such competitive interactions are unlikely to contribute to the early formation of a peripherally dependent pattern.

A third possibility is suggested by the report of Hamori et al. (1986) that the number of thalamic projection neurons in the vibrissae-related region of the ventroposterior nucleus is increased after neonatal damage to the vibrissae follicles. The possibility that a uniform increase in the number of thalamocortical projection neurons causes the lower density and in- 

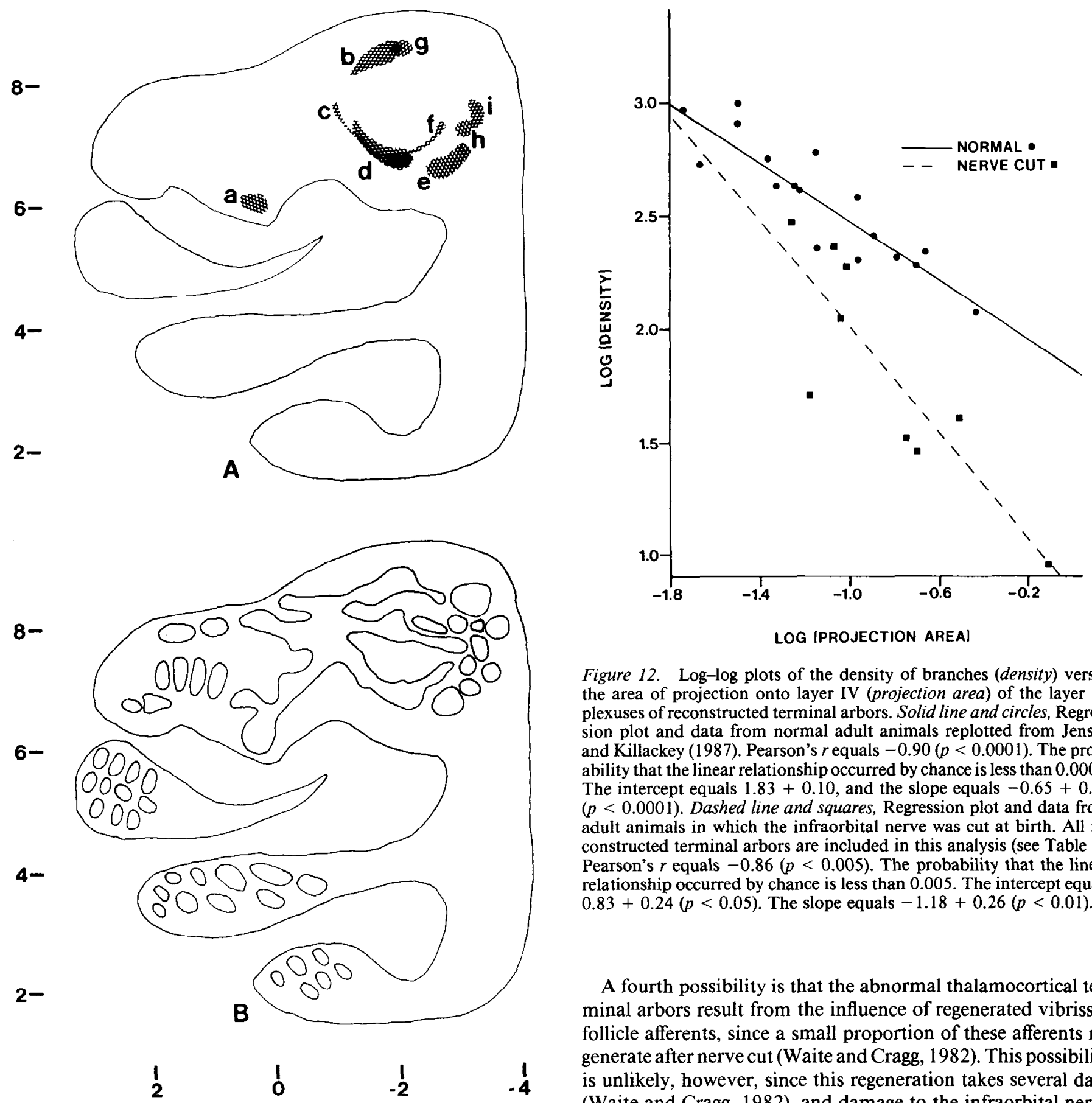

Figure 11. A, Diagrammatic representation of the approximate size and location of the layer IV plexuses of terminal arbors represented in the previous figures. $a$. Field corresponding to the terminal arbor in Figure $7 ; b$, to that in Figure $9 ; c$, Figure $3 ; d$, Figure 8 ; $e$, Figure $4 ; f$. Figure $10 ; g$, Figure $5, h$ and $i$, Fields corresponding to adjacent arbors in Figure 6. $B$, Diagrammatic representation of the pattern of thalamocortical projections from the ventroposterior nucleus, for comparison with $A$. The horizontal scale indicates millimeters from bregma; the vertical scale indicates millimeters lateral from the midline.

creased size of individual terminal arbors is unlikely, however, since an early uniform reduction in the number of vibrissae follicle afferents does not alter the size of vibrissae-related clusters in the cortex (Sikich et al., 1986).

Figure 12. Log-log plots of the density of branches (density) versus the area of projection onto layer IV (projection area) of the layer IV plexuses of reconstructed terminal arbors. Solid line and circles, Regression plot and data from normal adult animals replotted from Jensen and Killackey (1987). Pearson's $r$ equals $-0.90(p<0.0001)$. The probability that the linear relationship occurred by chance is less than 0.0001 . The intercept equals $1.83+0.10$, and the slope equals $-0.65+0.08$ $(p<0.0001)$. Dashed line and squares, Regression plot and data from adult animals in which the infraorbital nerve was cut at birth. All reconstructed terminal arbors are included in this analysis (see Table 1). Pearson's $r$ equals $-0.86(p<0.005)$. The probability that the linear relationship occurred by chance is less than 0.005 . The intercept equals $0.83+0.24(p<0.05)$. The slope equals $-1.18+0.26(p<0.01)$.

A fourth possibility is that the abnormal thalamocortical terminal arbors result from the influence of regenerated vibrissae follicle afferents, since a small proportion of these afferents regenerate after nerve cut (Waite and Cragg, 1982). This possibility is unlikely, however, since this regeneration takes several days (Waite and Cragg, 1982), and damage to the infraorbital nerve after postnatal day 4 does not dramatically alter the vibrissarelated clusters within the neocortex (Belford and Killackey, 1980; Bates and Killackey, 1985).

In summary, neonatal damage to the infraorbital nerve alters the somatotopic organization of thalamocortical projections to the somatosensory neocortex. A number of characteristics of the altered pattern can be attributed to changes in the morphology of individual terminal arbors. The size, complexity, and degree of overlap of individual terminal arbors appear to determine the overall pattern of the altered neocortical vibrissae-related map. We interpret these results as indicating that the influence of the periphery on the fine-grain organization of the neocortex is expressed in the morphology of individual thalamocortical axons. 


\section{References}

Bates, C. A., and H. P. Killackey (1985) The organization of the neonatal rat's brainstem trigeminal complex and its role in the formation of central trigeminal patterns. J. Comp. Neurol. 240: 265287.

Bates, C. A., R. S. Erzurumlu, and H. P. Killackey (1982) Central correlates of peripheral pattern alterations in the trigeminal system of the rat. III. Neurons of the principal sensory nucleus. Dev. Brain Res. 5: 108-113.

Belford, G. R., and H. P. Killackey (1980) The sensitive period in the development of the trigeminal system of the neonate rat. J. Comp. Neurol, 193: 335-350.

Dawson, D. R., and H. P. Killackey (1985) Distinguishing topography and somatotopy in the thalamocortical projections of the developing rat. Dev. Brain Res. 17: 309-313.

Durham, D., and T. A. Woolsey (1984) Effects of neonatal whisker lesions on mouse central trigeminal pathways. J. Comp. Neurol. 223: 424-447.

Erzurumlu, R. S., and H. P. Killackey (1983) Development of order in the rat trigeminal system. J. Comp. Neurol. 213: 365-380.

Hamori, J., C. Savy, M. Madarasz, J. Somogyi, J. Takacs, R. Verley, and E. Farkas-Bargeton (1986) Morphological alterations in subcortical vibrissal relays following vibrissal follicle destruction at birth in the mouse. J. Comp. Neurol. 254: 166-183.

Jacquin, M. F., and R. W. Rhoades (1985) Effects of neonatal infraorbital lesions upon central trigeminal primary afferent projections in rat and hamster. J. Comp. Neurol. 235: 129-143.

Jensen, K. F., and H. P. Killackey (1987) Terminal arbors of axons projecting to the somatosensory cortex of the adult rat. I. The normal morphology of specific thalamocortical afferents. J. Neurosci. 7: 35293543.

Killackey, H. P., and G. R. Belford (1979) The formation of afferent patterns in the somatosensory cortex of the neonatal rat. J. Comp. Neurol. 183: 285-304

Killackey, H. P., and G. R. Belford (1980) Central correlates of peripheral pattern alterations in the trigeminal system of the rat. Brain Res. 183: 205-210.

Killackey, H. P., and A. Shinder (1981) Central correlates of peripheral pattern alterations in the trigeminal system of the rat. II. The effect of nerve section. Dev. Brain Res. $1: 121-126$.

Killackey, H. P., G. Belford, R. Ruygo, and D. K. Ruygo (1976) Anomalous organization of thalamocortical projections consequent to vi- brissae removal in the newborn rat and mouse. Brain Res. 194: 309315.

Koralek, K. A., K. F. Jensen, and H. P. Killackey (1985) Projections of the medial portion of the posterior thalamic nuclear group (POM) to the somatosensory cortex of the rat. Soc. Neurosci. Abstr. 11:905.

Levay, S., M. P. Stryker, and C. J. Shatz (1978) Ocular dominance columns and their development in layer IV of the cat's visual cortex: A quantitative study. J. Comp. Neurol. 179: 223-244.

Levay, S., T. N. Weisel, and D. H. Hubel (1980) The development of ocular dominance columns in normal and visually deprived monkeys. J. Comp. Neurol. 191: 1-51.

Robson, J. A., C. A. Mason, and R. W. Guillery (1978) Terminal arbors of axons that have formed abnormal connections. Science 201 : 635-637.

Rosene, D. L., and M.-M. Mesulam (1978) Fixation variables in horseradish peroxidase neurohistochemistry. I. The effects of fixation time and perfusion procedures upon enzyme activity. J. Histochem. Cytochem. 26: 28-39.

Sikich, L., T. A. Woolsey, and E. M. Johnson, Jr. (1986) Effect of a uniform partial denervation of the periphery on the peripheral and central vibrissal system in guinea pigs. J. Neurosci. 6: 1227-1240.

Sretavan, D., and C. J. Shatz (1984) Prenatal development of individual retinogeniculate axons during the period of segregation. Nature 308: 845-848.

Sretavan, D., and C. J. Shatz (1986) Prenatal development of cat retinogeniculate axon arbors in the absence of binocular interactions. J. Neurosci. 6: 990-1003.

Sur, M., R. E. Weller, and S. M. Sherman (1984) Development of $\mathrm{X}$-and $\mathrm{Y}$-cell retinogeniculate terminations in kittens. Nature 310 : $246-248$.

Van der Loos, H., and T. A. Woolsey (1973) Somatosensory cortex: Structural alterations following early injury to sense organs. Science 179: 395-398.

Waite, P. M. E. (1984) Rearrangement of neuronal responses in the trigeminal system of the rat following peripheral nerve section. $J$. Physiol. (Lond.) 352: 425-445.

Waite, P. M. E., and B. G. Cragg (1982) The peripheral and central changes resulting from cutting or crushing the afferent nerve supply to the whiskers. Proc. R. Soc. Lond. [Biol.] 214: 191-211.

Wise, S. P., and E. G. Jones (1978) Developmental studies of thalamocortical and commissural connections in the rat somatic sensory cortex. J. Comp. Neurol. 178: 187-208. 